JOURNAL OF SYNCHROTRON RADIATION

ISSN 1600-5775

Received 2 April 2018

Accepted 10 October 2018

Edited by M. Eriksson, Lund University, Sweden

Keywords: microwave undulator; free-electron laser; corrugated waveguide; balanced hybrid condition.

\section{Systematic study of a corrugated waveguide as a microwave undulator}

\author{
Liang Zhang, ${ }^{a, b *}$ Wenlong He, ${ }^{a}$ Jim Clarke, ${ }^{b, c}$ Kevin Ronald, ${ }^{a}$ Alan D. R. Phelps ${ }^{a}$ and \\ Adrian Cross $^{a, b}$
}

\begin{abstract}
${ }^{\mathbf{a}}$ Department of Physics, SUPA, University of Strathclyde, Glasgow G4 ONG, UK, ${ }^{\mathbf{b}}$ The Cockcroft Institute, Sci-Tech Daresbury, Keckwick Lane, Daresbury, Warrington WA4 4AD, UK, and ${ }^{\mathrm{C}}$ ASTeC, STFC Daresbury Laboratory, Sci-Tech Daresbury, Keckwick Lane, Daresbury, Warrington WA4 4AD, UK. *Correspondence e-mail: liang.zhang@strath.ac.uk
\end{abstract}

Microwave undulators have great potential to be used in short-wavelength freeelectron lasers. In this paper, the properties of a corrugated waveguide and its performance as an undulator cavity for a UK X-ray free-electron laser were systematically studied. The equations presented in this paper allow a fast estimation of the dimensions of the corrugated waveguide. An undulator cavity operating at $36 \mathrm{GHz}$ designed for the $\mathrm{HE}_{11}$ and $\mathrm{HE}_{12}$ modes was investigated and the performance of both modes compared.

\section{Introduction}

Undulators are one of the most important components in a free-electron laser (FEL) (Deacon et al., 1977; Huang \& Kim, 2007). Currently, the conventional permanent magnet undulator (PMU) plays the dominant role. Microwave undulators (MUs) (Shintake et al., 1982, 1983) that also have a periodic magnetic field can be potential undulators and have the following advantages. (i) Fast dynamic control of the polarization (Shumail \& Tantawi, 2016). (ii) Easy control of the field strength, which can be adjusted through the input microwave power, whereas, in a PMU, mechanical methods to adjust the magnet gap or the magnet period have to be used which can be complicated. (iii) It is challenging for a PMU to achieve short periods as the magnetic field strength would be significantly reduced. In contrast, for an MU the equivalent period is mainly determined by the wavelength of the electromagnetic wave, therefore a short period can be achieved if the MU operates at a higher frequency. (iv) The MU is essentially a metallic cavity and hence it is robust against damage by ionizing radiation near electron beam dump regions, as compared with the PMUs that are made of rareearth materials, which may be more susceptible to damage in harsh ionizing radiation environments.

However, since the concept of the microwave undulator was proposed in 1982 (Shintake et al., 1982), progress has taken longer than expected, mainly due to the limited availability of high-power microwave sources. In 1983 (Shintake et al., 1983), the first MU experiment was carried out and an equivalent magnetic field $B_{\mathrm{u}}$ of $0.045 \mathrm{~T}$ with an undulator parameter $K$ of 0.24 was achieved when driven by a $300 \mathrm{~kW}, 2.856 \mathrm{GHz}$ microwave source. A ridged rectangular cavity was used and a quality factor $Q$ of 7100 was measured. To achieve a similar performance to a state-of-the-art PMU, for example, $1.29 \mathrm{~T}$ for a $15 \mathrm{~mm}$-period PMU used in the Swiss-FEL at the Paul Scherrer Institute, the required driving power would need to be more than $20 \mathrm{MW}$ at $10.5 \mathrm{GHz}$ (assuming the same $Q$ factor 
of 7100 can be achieved for a similar structure scaled for operation at $10.5 \mathrm{GHz}$ ). However, the electric field at the wall of the ridged rectangular cavity would be too high and susceptible to microwave breakdown. A significant improvement on the MU was made with the use of a low-loss $\mathrm{HE}_{11}$ mode in a corrugated waveguide. A cavity made of a corrugated waveguide operating at the $\mathrm{X}$-band was measured and it was able to achieve a $Q$ factor as high as 91000 . When it was driven by a $50 \mathrm{MW}$ SLAC klystron at $11.424 \mathrm{GHz}$ (Tantawi et al., 2014), such an MU was able to achieve an equivalent $B_{\mathrm{u}}$ of $0.65 \mathrm{~T}$ with a period of $13.9 \mathrm{~mm}$.

The corrugated waveguide is normally overmoded. The numerical calculation of the eigenmodes of the cavity made with the corrugated waveguide is time-consuming. In this paper, analytical equations and design constraints are summarized which could be used to quickly estimate the dimensions of the structure. The microwave undulator can be operated at a high millimetre-wave frequency to achieve a smaller period. In this paper, a corrugated waveguide operating at $36 \mathrm{GHz}$ was designed as an MU for a UK XFEL.

The paper is organized as follows. In Section 2, the principle of the MU and the relation between the cavity and the microwave sources is presented. Section 3 introduces the theory of the corrugated waveguide and the equations that determine the dimensions, field distribution and the loss coefficient. Section 4 details the simulation results of an MU operating at $36 \mathrm{GHz}$. Both the $\mathrm{HE}_{11}$ and $\mathrm{HE}_{12}$ operating modes were investigated and their performances compared.

\section{Principles of $\mathrm{MU}$}

The principle of the MU can be found in the literature (Pellegrini, 2006; Seidel, 2001; Shintake et al., 1983; Shumail \& Tantawi, 2016). The relativistic electrons in an MU cavity will interact with both the electric field $E_{x}=E_{0} \sin \left(2 \pi z / \lambda_{\mathrm{g}}\right) \sin (\omega t)$ and magnetic field $B_{y}=B_{0} \cos \left(2 \pi z / \lambda_{\mathrm{g}}\right) \cos (\omega t)$. Compared with a PMU, the Lorentz force in an MU can be rewritten in the form

$$
\begin{aligned}
F_{x}= & \frac{e E_{0}}{2}\left(\frac{\varsigma}{Z_{\mathrm{w}}}+1\right) \cos \left[2 \pi z\left(\frac{1}{\lambda_{0}}+\frac{1}{\lambda_{\mathrm{g}}}\right)\right] \\
& +\frac{e E_{0}}{2}\left(\frac{\varsigma}{Z_{\mathrm{w}}}-1\right) \cos \left[2 \pi z\left(\frac{1}{\lambda_{0}}-\frac{1}{\lambda_{\mathrm{g}}}\right)\right],
\end{aligned}
$$

where $e$ is the charge on the electron, $\lambda_{0}$ is the free space wavelength, and $\lambda_{\mathrm{g}}$ is the wavelength of the electromagnetic wave in the undulator cavity. $E_{0}$ and $B_{0}$ are the peak electric and magnetic field strength in the microwave undulator cavity, respectively. $Z_{\mathrm{w}}$ is the wave impedance in the cavity and $\zeta$ is the wave impedance in free space. The second term leads to a long wavelength and is undesirable in the undulator of a shortwavelength FEL. The second term can be ignored if the wave impedance is close to the free-space impedance, which means the operating frequency is far from the cut-off frequency of the waveguide. In this case, the equivalent magnetic field $B_{\mathrm{u}}$ and wavelength $\lambda_{\mathrm{u}}$ of the microwave undulator are given by

$$
\begin{aligned}
B_{\mathrm{u}} & =\frac{E_{0}}{2 c}\left(\frac{\varsigma}{Z_{\mathrm{w}}}+1\right), \\
\frac{1}{\lambda_{\mathrm{u}}} & =\frac{1}{\lambda_{0}}+\frac{1}{\lambda_{\mathrm{g}}} .
\end{aligned}
$$

If the microwave source starts to fill the cavity at time zero, the stored energy at time $t$ in the cavity can be expressed as (Alvarez, 1986)

$$
W(t)=P_{0} \tau_{0} \frac{4 \beta}{(1+\beta)^{2}}\left[1-\exp \left(-\frac{1+\beta}{2} \frac{t}{\tau_{0}}\right)\right]^{2},
$$

where $P_{0}$ is the input power, $\tau_{0}=Q_{0} / \omega$, and $\beta=Q_{0} / Q_{\mathrm{e}} \cdot Q_{0}$ and $Q_{\text {e }}$ represent the intrinsic and external quality factor of the cavity. In a steady state, where $t \gg \tau_{0}$, the stored energy reaches its maximum value of $P_{0} \tau_{0}$ if the coupling aperture is specifically designed to achieve $\beta=1$. The input power in this case will be equal to the Ohmic loss in the cavity. In the cases of an under-coupled $(\beta<1)$ and over-coupled $(\beta>1)$ regime, the stored energy in the steady state is smaller. The filling time of the cavity $t_{\mathrm{tf}}$ can be calculated if a charging factor $\eta$, defined as the ratio of the charged energy and its maximum value, is known,

$$
t_{\mathrm{tf}}=\frac{Q_{0}}{\omega} \frac{2}{1+\beta} \ln \left(\frac{1+\eta^{1 / 2}}{1-\eta}\right) .
$$

From equations (3) and (4), to achieve maximum storage at a given input power, a higher $Q$ factor of the cavity is preferred; however, the filling time increases simultaneously. In applications of MUs, high-power microwave sources with output powers of megawatts or more are needed to achieve a high equivalent magnetic field. Such high-power microwave sources normally operate in pulsed mode to reduce the power supply requirements and associated thermal stress. The filling time of the MU cavity should match the pulse length of the microwave source. The coupler can be slightly over-coupled to reduce the filling time while maintaining a high $Q$ factor. If the input microwave frequency is $36 \mathrm{GHz}$, with a $2 \mu$ s pulse length, the $Q$ factor of the cavity should be under 150000 if $\beta=1$ and $\eta=0.9$.

The electric field strength inside the cavity along the electron beam path can be calculated by careful selection of the operating mode of the cavity. Knowing the input power and $Q$ factor, the parameters of the MU can be determined.

\section{Corrugated waveguide}

A high- $Q$ low-loss cavity is of great importance when it is used as an MU. In a circular waveguide, the mode with the lowest loss is the $\mathrm{TE}_{01}$ mode. However, the field strength at the waveguide center for this mode is small and hence is not a good option for an MU as the electrons are to propagate down the center of the structure. A corrugated waveguide, as shown in Fig. 1, can be used in other applications such as a feed horn for a transmission line system due to its advantages of low cross-polarization field, low loss and wide bandwidth. Its low attenuation feature is attractive for the transmission of high- 


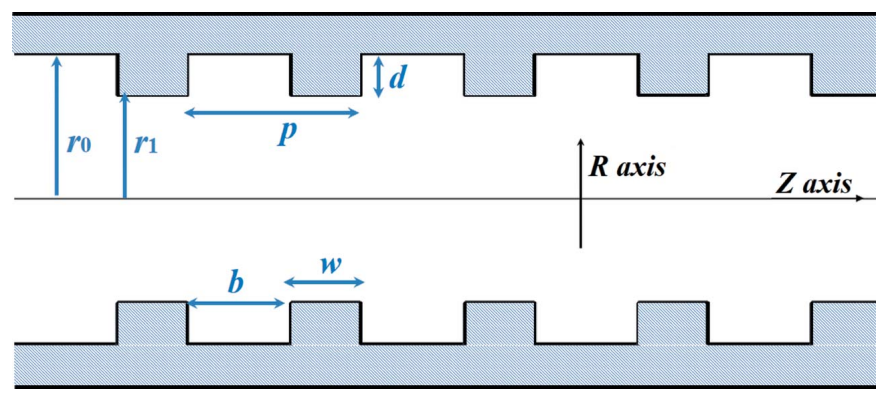

Figure 1

Schematic drawing of the corrugated waveguide.

power microwaves. The fundamental mode $\mathrm{HE}_{11}$ was found to have lower loss compared with the $\mathrm{TE}_{01}$ mode in the circular waveguide. It has been used to transport megawatt-level millimetre-wavelength radiation generated by gyrotron oscillators for fusion experiments such as ITER. In recent years, the corrugated waveguide has been proposed as an MU (Shumail et al., 2011; Chang et al., 2012; Toufexis \& Tantawi, 2017).

The corrugated waveguide contains circular waveguide steps and smooth sections. The properties of a corrugated waveguide with arbitrary radial corrugation depth can be accurately solved using a mode-matching method (James, 1981; Neilson et al., 1989; Zhang et al., 2016). For the periodically corrugated waveguide used for transmitting the microwave power, analytical equations can be derived based on the fact that the waveguide radius is larger than the wavelength. The propagation characteristics of the periodically corrugated waveguide were studied using the simplified surface-impedance approach or the rigid equations taking into account the spatial harmonics in the corrugation gaps (Clarricoats \& Olver, 1984; Dragone, 1980; Clarricoats \& Saha, 1971; Kowalski et al., 2010).

The surface-impedance approach assumes that only the lowest transverse magnetic (TM) standing wave exists in the slot and ignores its spatial harmonics. It gives a good approximation when the period per wavelength, defined by $\lambda_{0} / p$, is a reasonably large value and the corrugation slot length, defined by $w=p-b$ in Fig. 1 , is a small value. At low frequency, for example in the application of the microwave undulator, these assumptions would normally be satisfied. If only the lowest TM standing wave were present in the corrugation slot, its surface admittance at $r=r_{1}$ can be written as

$$
\frac{H_{\psi}}{E_{z}}=-j Y_{0} \frac{J_{m}^{\prime}\left(x_{1}^{\prime}\right) Y_{m}\left(x_{0}^{\prime}\right)-J_{m}\left(x_{0}^{\prime}\right) Y_{m}^{\prime}\left(x_{1}^{\prime}\right)}{J_{m}\left(x_{1}^{\prime}\right) Y_{m}\left(x_{0}^{\prime}\right)-J_{m}\left(x_{0}^{\prime}\right) Y_{m}\left(x_{1}^{\prime}\right)},
$$

where $m$ indicates the azimuthal mode number, $x_{1}^{\prime}=k r_{1}$ and $x_{0}^{\prime}=k r_{0} . J_{m}$ and $Y_{m}$ are the first and second kind of Bessel functions of order $m$, respectively. $Y_{0}$ is the free-space wave admittance. The surface admittance becomes 0 when

$$
J_{m}^{\prime}\left(x_{1}^{\prime}\right) Y_{m}\left(x_{0}^{\prime}\right)-J_{m}\left(x_{0}^{\prime}\right) Y_{m}^{\prime}\left(x_{1}^{\prime}\right)=0 .
$$

This is known as the balanced hybrid condition. If the operating frequency $(f)$ and the waveguide radius (either $r_{0}$ or $r_{1}$ ) are given, the corrugation depth $d=r_{0}-r_{1}$ can be determined from the equation. At $m=1$ and $x_{1}^{\prime} \gg 1$, the surface admittance can be further simplified as $H_{\psi} / E_{z}=j Y_{0} \cot (k d)$. The corrugation depth $d$ would be equal to $\lambda_{0} / 4$ to ensure $H_{\psi}=0$.

Under the balanced hybrid condition and $x_{1}^{\prime} \gg 1$, the dispersion curve between $k$ and $k_{z}$ is determined by

$$
K r_{1} \frac{J_{m}^{\prime}\left(K r_{1}\right)}{J_{m}\left(K r_{1}\right)}=\mp m \frac{k_{z}}{k},
$$

where $K^{2}=k^{2}-k_{z}^{2}$, the '-' in ' $\mp$ ' denotes the HE modes, and the ' + ' denotes the EH modes. For a large radius, which leads to $k_{z} \simeq k$ and $m=1$, equation (7) can be further simplified to $J_{0}\left(K r_{1}\right)=0$ for $\mathrm{HE}_{1 \mathrm{n}}$ modes and $J_{2}\left(K r_{1}\right)=0$ for $\mathrm{EH}_{1 n}$ modes (Shumail, 2014).

To describe the field distribution inside the corrugated waveguide, different eigenmode sets using TE/TM and $\mathrm{HE} / \mathrm{EH}$ combinations have been derived (Crenn \& Charollais, 1996). The field inside the corrugated waveguide with the linearly polarized mode sets can be simplified as (Clarricoats \& Saha, 1971)

$$
\begin{aligned}
& E_{z}=a_{m} J_{m}(K r) \cos (m \varphi), \\
& E_{r}=-j a_{m}(k / K) J_{m \mp 1}(K r) \cos (m \varphi), \\
& E_{\varphi}=\mp(-j) a_{m}(k / K) J_{m \mp 1}(K r) \sin (m \varphi) .
\end{aligned}
$$

Usually, a higher order $\mathrm{HE}$ or $\mathrm{EH}$ mode is not linearly polarized. The suitable operating modes in the corrugated waveguide for the microwave undulator application are the $\mathrm{HE}_{11}$ and $\mathrm{HE}_{12}$ modes because they are low loss, linearly polarized and have the peak electric field at the waveguide center. The electric field patterns of the $\mathrm{HE}_{11}$ and $\mathrm{HE}_{12}$ modes are shown in Fig. 2. The $\mathrm{HE}_{12}$ mode has larger field density at the waveguide center. It can have a bigger electric field compared with the $\mathrm{HE}_{11}$ mode at the same input power.

The attenuation coefficient defined by the ratio between the lost power and the transported power per meter for the $\mathrm{HE}_{1 n}$ modes, under the balanced hybrid condition, can be written as (Clarricoats \& Olver, 1984; Doane, 1985)

$$
\alpha=\frac{1}{2}\left(\frac{R_{\mathrm{s}}}{Z_{0}}\right) \frac{x_{1}^{2} k}{x_{1}^{\prime 4}}\left[\frac{1}{k b}\left(1+\frac{x_{1}^{\prime} \pi}{2}\right)+x_{1}^{\prime}\right],
$$

where $Z_{0}=1 / Y_{0}$ is the free space wave impedance, $R_{\mathrm{s}}=$ $\left(\pi f \mu_{0} \sigma\right)^{1 / 2}$ is the resistivity of the corrugated metal waveguide and $\sigma$ is the conductivity of the metal. In this paper, oxygenfree high-conductivity copper was chosen and $\sigma=5.8 \times$ $10^{7} \mathrm{~S} \mathrm{~m}^{-1}$ was used in the simulation. Equation (9) implies that the attenuation coefficient is proportional to $r_{1}^{-3}$ and $f^{-2}$ in the case of $x_{1}^{\prime} \gg 1$.

The corrugated waveguide can be shortened at both ends to form a cavity. The resonance frequency of the corrugated waveguide can be estimated as

$$
f=\frac{c}{\lambda_{0}}=\frac{c}{2 \pi}\left[K^{2}+\left(\frac{N \pi}{\lambda_{\mathrm{g}}}\right)^{2}\right]^{1 / 2},
$$

where $c$ is the speed of light, $N$ is an integer and $\lambda_{\mathrm{g}}$ is the wavelength of the resonance mode in the cavity. Because the 


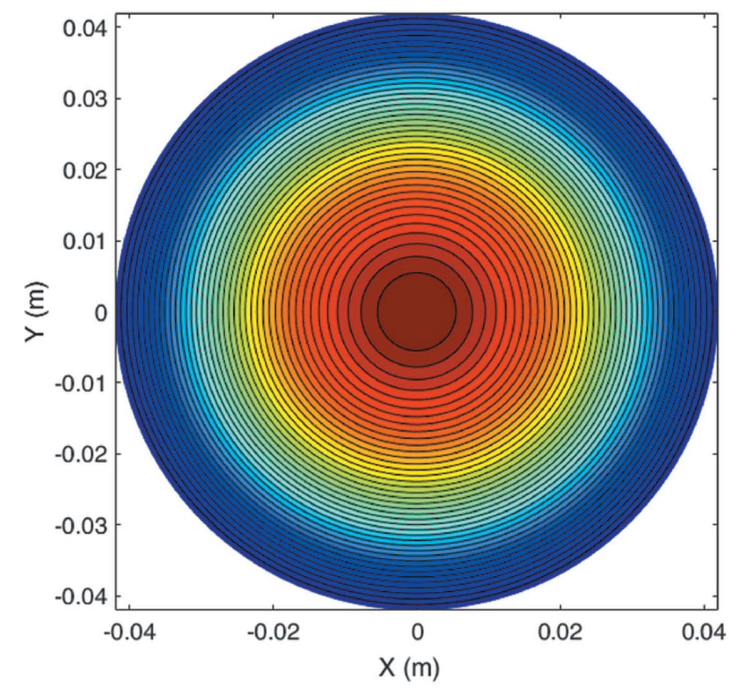

(a)

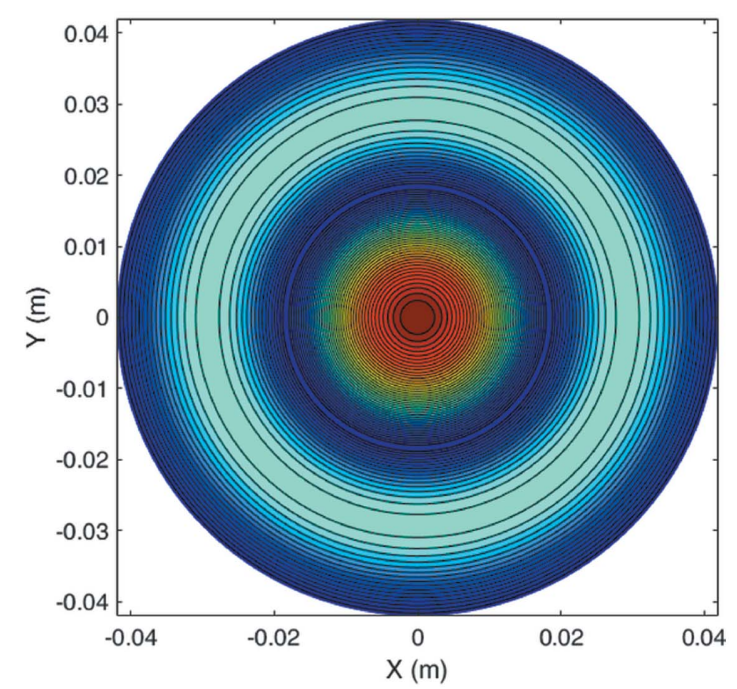

(b)

Figure 2

Contour plot of the electric field patterns of the $(a) \mathrm{HE}_{11}$ and $(b) \mathrm{HE}_{12}$ modes.

corrugated waveguide is normally overmoded, $\lambda_{0} \simeq \lambda_{\mathrm{g}}$ when $N=2$. As mentioned earlier, the surface-impedance approach gives a good approximation when $\lambda_{0} / p$ is a reasonably large value. Therefore, when designing the corrugated waveguide, the period $p$ can be selected to be smaller than $\lambda_{0} / 2$. It is of course the case that 'the smaller the period, the better'; however, as the operating frequency increases, the wavelength becomes smaller, resulting in a small corrugation period and a thin corrugation slot of $w \simeq 0$ which significantly increases the machining difficulty. The final choice of the geometry should therefore also consider the machining tolerance.

Since $x_{1}^{\prime}=k r_{1}=2 \pi r_{1} / \lambda_{0} \gg 1$ has to be satisfied, the corrugated waveguide radius $r_{1}$ is a large value. As the attenuation coefficient is proportional to $r_{1}^{-3}$, it is preferable to have a large $r_{1}$ at a given operating frequency. From equation (8), the waveguide radius affects the field strength if the input power is a fixed value. In a microwave undulator, a high field at the electron beam path, in this case the waveguide center, is
Table 1

Parameters of the MU composed of a corrugated waveguide.

\begin{tabular}{lll}
\hline Operating mode & $\mathrm{HE}_{11}$ & $\mathrm{HE}_{12}$ \\
\hline Operating frequency $(\mathrm{GHz})$ & 36 & 36 \\
$\lambda_{0}(\mathrm{~mm})$ & 8.33 & 8.33 \\
$R_{\mathrm{b}}(\mathrm{mm})$ & 2.0 & 2.0 \\
$r_{1}(\mathrm{~mm})$ & $4 R_{\mathrm{b}}=8.0$ & $9 R_{\mathrm{b}}=18.0$ \\
$d=\lambda_{0} / 4(\mathrm{~mm})$ & 2.1 & 2.1 \\
$\lambda_{\mathrm{g}}(\mathrm{mm})$ & 9.06 & 9.12 \\
$p=\lambda_{\mathrm{g}} / 3(\mathrm{~mm})$ & 3.00 & 3.02 \\
$w(\mathrm{~mm})$ & 0.5 & 0.5 \\
$b=p-w(\mathrm{~mm})$ & 2.50 & 2.52 \\
$Q$ factor & 94344 & 187073 \\
Input power $(\mathrm{MW})$ & 50 & 50 \\
Peak $E x$ on axis $\left(\mathrm{V} \mathrm{m}^{-1}\right)$ & $3.8 \times 10^{8}$ & $3.7 \times 10^{8}$ \\
$B_{\mathrm{u}}(\mathrm{T})$ & 1.27 & 1.23 \\
$\lambda_{\mathrm{u}}(\mathrm{mm})$ & 4.34 & 4.35 \\
$K_{\mathrm{u}}$ & 0.52 & 0.50 \\
\hline
\end{tabular}

desired. Therefore $r_{1}$ should be as small as possible under the constraint of $k r_{1} \gg 1$. On the other hand, it is preferred for the electron beam that travels through the microwave undulator to see a uniform field in the radial direction. The minimum waveguide radius can be solved from the field pattern of the operating mode if the electron beam aperture $R_{\mathrm{b}}$ and a threshold, for example $90 \%$ of the maximum field at the beam edge, are defined. For $\mathrm{HE}_{1 n}$ modes, it follows that $J_{1}\left(K R_{\mathrm{b}}\right)=$ 0.9. Taking the first two solutions of $J_{0}\left(K r_{1}\right)=0, K$ is approximately equal to $2.4 / r_{1}$ or $5.5 / r_{1}$ for the $\mathrm{HE}_{11}$ or the $\mathrm{HE}_{12}$ mode, respectively. Therefore the following parameters were chosen,

$$
r_{1}= \begin{cases}3.8 R_{\mathrm{b}}, & \text { for the } \mathrm{HE}_{11} \text { mode, } \\ 8.6 R_{\mathrm{b}}, & \text { for the } \mathrm{HE}_{12} \text { mode }\end{cases}
$$

resulting in a reasonable value of $r_{1}$. Meanwhile the value of $r_{1}$ should satisfy $x_{1}^{\prime}=2 \pi r_{1} / \lambda_{0} \gg 1$. If $x_{1}^{\prime}=5$ is used, then $r_{1} \geq 0.8 \lambda_{0}$ and the results become

$$
r_{1}= \begin{cases}\operatorname{maxima}\left(3.8 R_{\mathrm{b}}, 0.8 \lambda_{0}\right), & \text { for the } \mathrm{HE}_{11} \text { mode } \\ \operatorname{maxima}\left(8.6 R_{\mathrm{b}}, 0.8 \lambda_{0}\right), & \text { for the } \mathrm{HE}_{12} \text { mode }\end{cases}
$$

\section{Design of a corrugated waveguide for an MU}

A corrugated waveguide cavity operating at $36 \mathrm{GHz}$ was designed as a potential microwave undulator for the UK XFEL. The initial geometry parameters were calculated from the equations described in previous sections. Both of the $\mathrm{HE}_{11}$ and $\mathrm{HE}_{12}$ modes are considered. A summary of the geometry parameters as well as the undulator deflection parameter $K_{\mathrm{u}}=0.09336 B_{\mathrm{u}} \lambda_{\mathrm{u}}[\mathrm{T} \mathrm{mm}]$ are listed in Table 1.

The accurate resonance frequency and $Q$ factor of the designed structure were simulated using CST Microwave Studio. The simulated structure was set at six corrugation periods to significantly reduce the simulation time. For such a short cavity length, the loss at the conducting wall at both sides will be much larger than the loss at the corrugated wall because the field strength at the corrugated wall is much smaller. In the simulation, a periodic boundary condition was set at both ends of the corrugated waveguide. In this way, the 

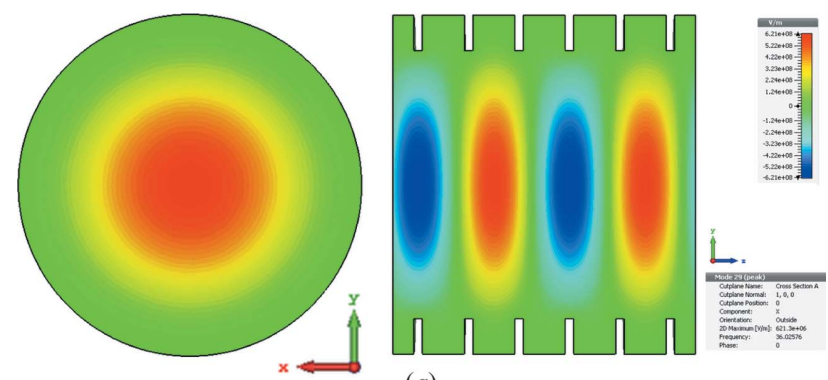

(a)
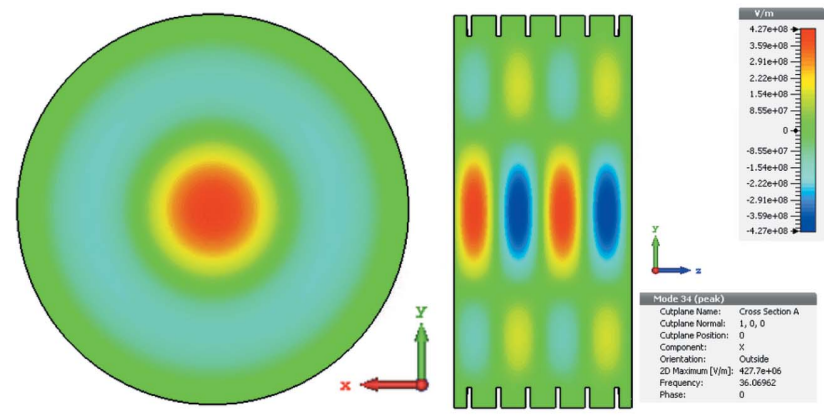

(b)

Figure 3

Electric field patterns of the $(a) \mathrm{HE}_{11}$ and $(b) \mathrm{HE}_{12}$ modes.

loss at both sides will not be included. Fig. 3 shows the electric field distribution of the cavity modes of $\mathrm{HE}_{114}$ and $\mathrm{HE}_{124}$. The peak $E x$ field along the $z$ axis was obtained from the field distribution of the eigenmode and normalized to an Ohmic loss power of $50 \mathrm{MW}$ at a cavity length of $1 \mathrm{~m}$. With the same input power and similar operating frequency, the waveguide radius of the $\mathrm{HE}_{12}$ mode was about 2.2 times that of the $\mathrm{HE}_{11}$ mode, and its $Q$ factor was nearly 2 times that of the $\mathrm{HE}_{11}$ mode. The $E x$ field strength was slightly smaller than that of the $\mathrm{HE}_{11}$ mode. The equivalent magnetic field $B_{\mathrm{u}}$ was also similar for both cases.

Parameter scans were used to study the effects of varying of the corrugation geometry parameters such as the corrugation period $(p)$, the slot length $(w)$ and the waveguide radius $\left(r_{1}\right)$. The results of resonance frequency, $Q$ factor and equivalent magnetic field strength are shown in Figs. 4-6. The $\mathrm{HE}_{11}$ mode has a similar trend to the $\mathrm{HE}_{12}$ mode. In Fig. 4, as the corrugation period increases, the resonance frequency becomes smaller while the slot length $(w)$ and the waveguide radius $\left(r_{1}\right)$ are kept the same. The results matched the theoretical calculation well from equation (10) and the scaling law equations (Shumail et al., 2011). The difference between the simulation and theoretical calculation is less than $2 \%$, therefore it allows a fast prediction of the cavity parameters from the theoretical analysis. The $Q$ factor reduces as the corrugation period increases. It is mainly caused by the increase of the overall waveguide length and the decreases in the stored energy per unit length.

The simulation results at the different waveguide radii are shown in Fig. 5. The trends for the $\mathrm{HE}_{11}$ and $\mathrm{HE}_{12}$ modes are very similar. Increasing the waveguide radius would significantly increase the $Q$ factor and the resonance frequency drops quickly. However, from equation (4), a high $Q$ factor would result in a long filling time. For the $\mathrm{HE}_{11}$ mode, a $20 \%$ increment of the waveguide radius would lead to a $Q$ factor close to 150000 , requiring an increase in the pulse length of the high-power microwave source used to drive the structure. Also, increasing the waveguide radius did not improve the equivalent magnetic field, which was almost the same. Therefore, the designed waveguide radius was considered to be optimal.

The simulation results of the variation in slot length while keeping the corrugation period constant are shown in Fig. 6. Only the CST simulation results were included as the theoretical analysis will not be accurate at small slot length values. As the slot length increases, the assumption of only the lowest TM standing wave appearing in the slot is no longer satisfied. The field at the corrugation wall became larger and caused a significant reduction in the cavity $Q$. The $Q$ factor dropped more quickly for the $\mathrm{HE}_{11}$ mode than the $\mathrm{HE}_{12}$ mode because its waveguide radius was smaller. As the corrugation period was kept constant, the change in the resonance frequency was small. The equivalent magnetic field $B_{\mathrm{u}}$ also changed by a small amount. The simulation results proved that the slot length should be as small as possible to achieve a high $Q$

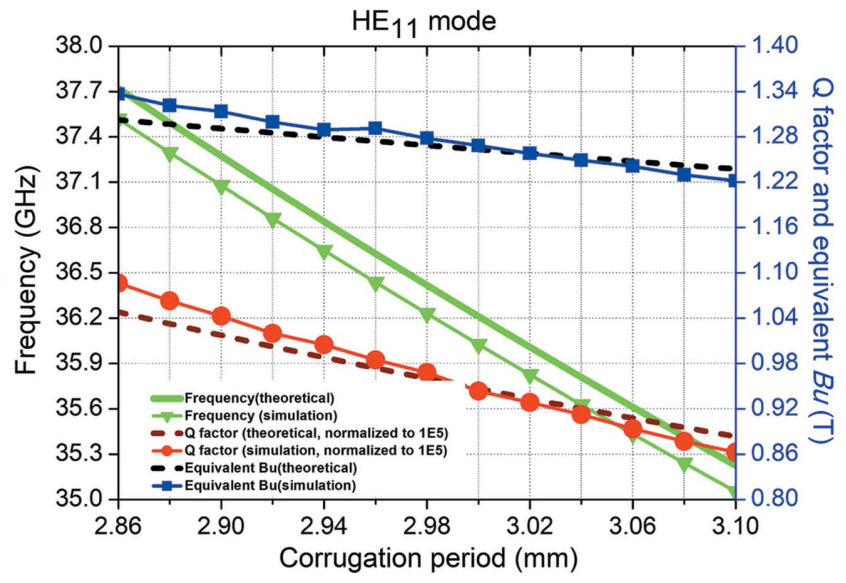

(a)

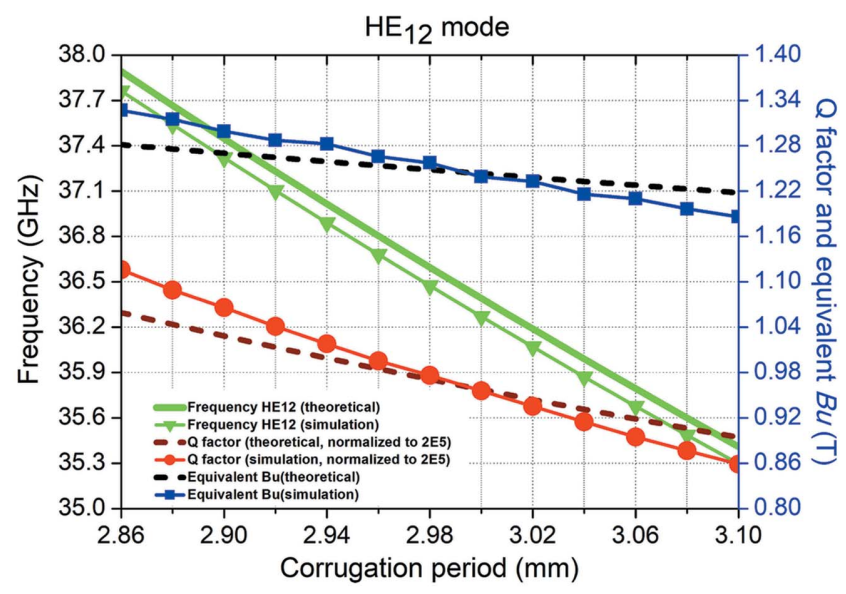

(b)

Figure 4

Simulation results of different corrugation periods: $(a) \mathrm{HE}_{11}$ mode and (b) $\mathrm{HE}_{12}$ mode. 


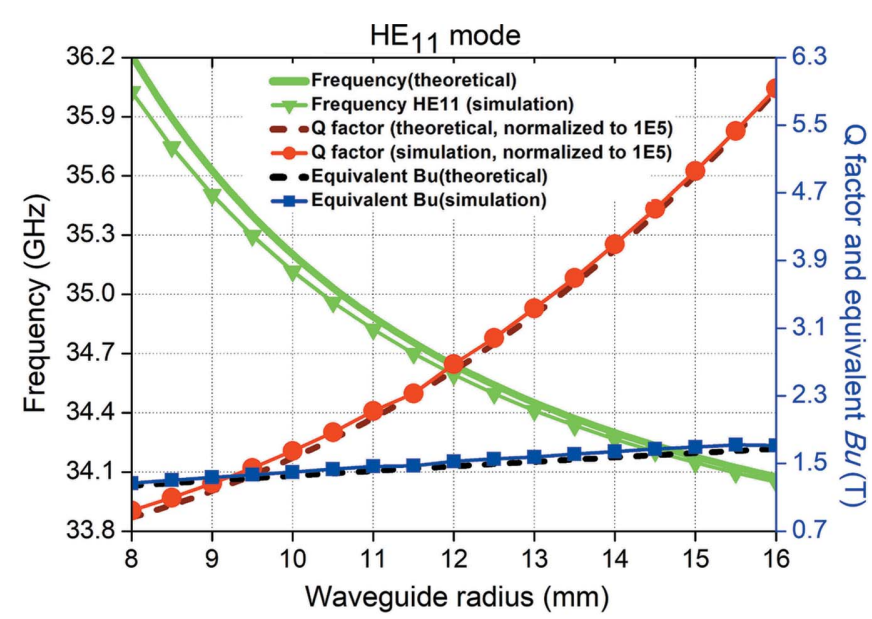

(a)

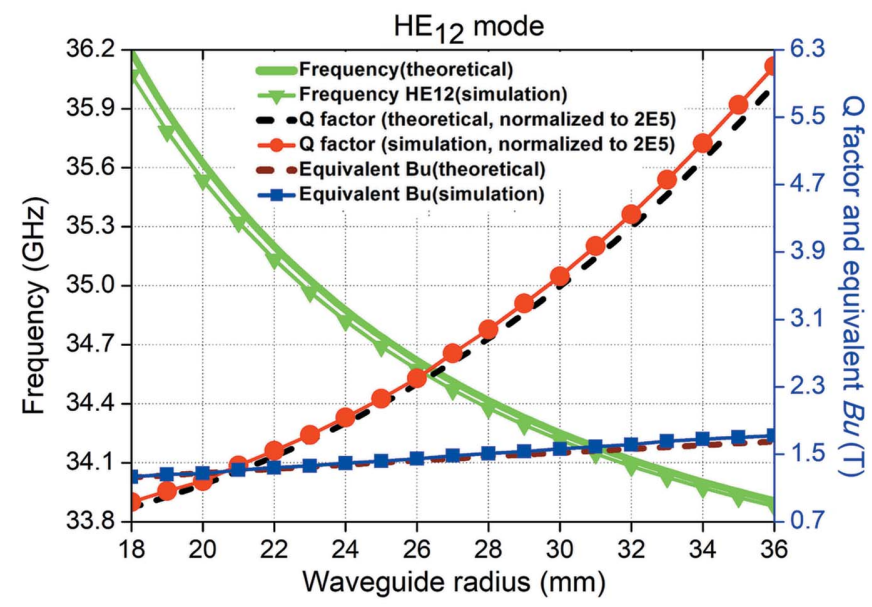

(b)

Figure 5

Simulation results of different waveguide radii: (a) $\mathrm{HE}_{11}$ mode and $(b)$ $\mathrm{HE}_{12}$ mode.

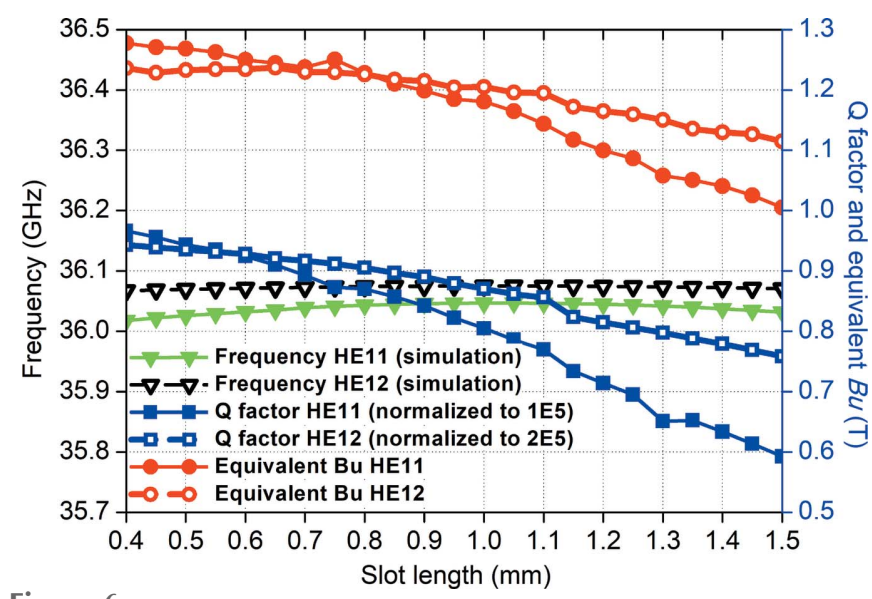

Figure 6

Simulation results of different slot lengths.

factor. However, the difficulty in manufacturing such a structure while maintaining sufficient mechanical strength needs to be taken into account. A slot length of $0.5 \mathrm{~mm}$ is a reasonable value to satisfy the criteria of ease of manufacture, while at the same time being mechanically strong.

\section{Discussion and conclusions}

In this paper, a corrugated waveguide was studied as a microwave undulator for a UK XFEL. The equations that govern the performance of the microwave undulator are presented and have been used to estimate the dimensions of the corrugated waveguide.

Both of the $\mathrm{HE}_{11}$ and $\mathrm{HE}_{12}$ modes existing in the corrugated waveguide are suitable operating modes. Their characteristics were studied using numerical simulation and their performance compared in the design of a corrugated waveguide operating at $36 \mathrm{GHz}$. It was found that the equivalent magnetic field for both of the modes was very similar. The $\mathrm{HE}_{12}$ mode had a much larger waveguide radius, which could be advantageous for high-frequency operation because it helps to reduce the difficulty in manufacture as well as reducing the electric field at the wall. The $\mathrm{HE}_{12}$ mode is also less sensitive to the slot length compared with the $\mathrm{HE}_{11}$ mode. The drawback of the $\mathrm{HE}_{12}$ mode includes a much higher $Q$ factor that leads to a longer filling time and greater sensitivity to the waveguide period.

\section{Funding information}

The authors would like to thank the Science and Technology Facilities Council (STFC) UK and the Cockcroft Institute (Core Grant ST/P002056/1) for supporting this work.

\section{References}

Alvarez, R. A. (1986). Rev. Sci. Instrum. 57, 2481-2488.

Chang, C., Shumail, M., Tantawi, S., Neilson, J. \& Pellegrini, C. (2012). Appl. Phys. Lett. 101, 161102.

Clarricoats, P. J. B. \& Olver, A. D. (1984). Corrugated Horns for Microwave Antennas, IEE Electromagnetic Waves Series, Vol. 18. Institution of Engineering and Technology.

Clarricoats, P. J. B. \& Saha, P. K. (1971). Proc. Inst. Electr. Eng. UK, 118, 1177-1186.

Crenn, J. P. \& Charollais, C. (1996). Int. J. Infrared Milli Waves, 17, 1475-1506.

Deacon, D. A. G., Elias, L. R., Madey, J. M. J., Ramian, G. J., Schwettman, H. A. \& Smith, T. I. (1977). Phys. Rev. Lett. 38, 892894.

Doane, J. L. (1985). Infrared Millimeter Waves, Vol. 13, pp. 123-170. Orlando: Academic Press.

Dragone, C. (1980). IEEE Trans. Microw. Theory Techn. 28, 704-710.

Huang, Z. \& Kim, K.-J. (2007). Phys. Rev. ST Accel. Beams, 10, 034801.

James, G. L. (1981). IEEE Trans. Microw. Theory Techn. 29, 10591066.

Kowalski, E. J., Tax, D. S., Shapiro, M. A., Sirigiri, J. R., Temkin, R. J., Bigelow, T. S. \& Rasmussen, D. A. (2010). IEEE Trans. Microw. Theory Techn. 58, 2772-2780.

Neilson, J. M., Latham, P. E., Caplan, M. \& Lawson, W. G. (1989). IEEE Trans. Microw. Theory Techn. 37, 1165-1170.

Pellegrini, C. (2006). AIP Conf. Proc. 807, 30-45.

Seidel, M. (2001). TESLA-FEL 2001-08, pp. 1-14. TESLA-FEL Reports, DESY, Hamburg, Germany.

Shintake, T., Huke, K., Tanaka, J., Sato, I. \& Kumabe, I. (1982). Jpn. J. Appl. Phys. 21, L601-L603.

Shintake, T., Huke, K., Tanaka, J., Sato, I. \& Kumabe, I. (1983). Jpn. J. Appl. Phys. 22, 844-851. 
Shumail, M. (2014). PhD thesis, Stanford University, USA.

Shumail, M., Bowden, G. B., Chang, C., Neilson, J., Tantawi, S. G. \& Pellegrini, C. (2011). Proceedings of the 2nd International Particle Accelerator Conference (IPAC 2011), 4-9 September 2011, San Sebastian, Spain, pp. 3326-3328. THPC183.

Shumail, M. \& Tantawi, S. G. (2016). Phys. Rev. ST Accel. Beams, 19, 074001.
Tantawi, S., Shumail, M., Neilson, J., Bowden, G., Chang, C., Hemsing, E. \& Dunning, M. (2014). Phys. Rev. Lett. 112, 164802.

Toufexis, F. \& Tantawi, S. (2017). Proceedings of the 8th International Particle Accelerator Conference (IPAC 2017), 14-19 May 2017, Copenhagen, Denmark, pp. 1643-1646. TUPAB135.

Zhang, L., He, W., Donaldson, C. R. \& Cross, A. W. (2016). J. Infrared, Millim. Terahertz Waves, 37, 846-856. 